\title{
On the Synthesis of Control Laws for a Network of Autonomous Agents
}

\author{
Vijay Gupta, Babak Hassibi and Richard M Murray
}

\begin{abstract}
We study the synthesis problem of a LQR controller when the matrix describing the control law is additionally constrained to lie in a particular vector space. Our motivation is the use of such control laws to stabilize networks of autonomous agents in a decentralized fashion; with the information flow being dictated by the constraints of a pre-specified topology. We formulate the problem as an optimization problem and provide numerical procedures to solve it. Then we apply the technique to the decentralized vehicle formation control problem and show that the topology can have a significant effect on the optimal cost.
\end{abstract}

\section{INTRODUCTION AND MOTIVATION}

Control of dynamic agents coupled to each other through an information flow network has emerged as a topic of major interest in recent years. Such a setting can be used to model many real-life situations, such as air traffic control, satellite clusters, swarms of robots, UAV formations, and potentially such applications as the Internet. Compared with the more traditional applications of control theory, there are fundamentally new features introduced in this problem. The topology of the information network can have many effects. On one hand, it might introduce instability if the information being fed through the network adds on constructively to the disturbance at a node (see, e.g, [8], [12], [10]); on the other, intuitively, it should serve as a means for better noise rejection for the network as a whole.

However, most of the work on the problem so far has centered on stability analysis of the formation assuming certain control laws in place. A more general question is that of synthesis of the control law to be used by the agents in such a formation, such that some cost function is optimized. The defining feature of the problem is that while the cost function can involve all the individual agents in the formation; the pre-specified topology of the formation imposes constraints on the form of the control law by limiting the information available to various agents at any time. Thus, it is not realistic to assume that an agent would know the state of all the other agents in the formation at any given time and be able to use it to calculate the control input. These features make the problem a decentralized control problem with arbitrary information flow patterns, which is, in general, much harder to solve than the traditional optimal control problem.

Research in decentralized control has a long history. Witsenhausen [23], [24] showed that under the decentral-

\footnotetext{
Division of Engineering and Applied Science, California Institute of Technology, Pasadena, CA 91125, USA \{gupta, hassibi, murray\}ecaltech.edu.

Work supported in part by AFOSR grant F49620-01-1-0460.
}

ized information constraints, a linear controller might not be optimal and also that the cost function need not be convex in the controller variables. A discrete equivalent of Witsenhausen's counter-example was given in [18] where it was also shown that the problem of finding a stabilizing controller under the information pattern constraints is NPcomplete. For particular information structures, the problem has been solved, e.g., see [6]. Some researchers have also studied this problem under the assumption of spatial invariance by using a multidimensional approach [1], [3], [4]. Rotkowitz et al. [19] gave certain invariance conditions under which the problem retains the convex character. A different approach for solving the problem was inspired by the design of reduced-order controllers (e.g. [16]) and yielded numerical algorithms for solving the optimal linear control with arbitrary number of free parameters [20], [22]. The vehicle formation problem was considered in [5] where the $H_{2}$ synthesis problem was posed as an optimization problem and a sub-optimal solution presented.

In this paper, we set up the LQR problem for the problem of controlling a network of autonomous agents with a given information flow topology. Even if the dynamics of the agents are not coupled and the only coupling present is due to the cost function; the optimal control law, in general, requires every agent to use knowledge about every other agent. We impose a pre-specified topology on the form of the control law which specifies the set of agents with which a particular agent can communicate; and solve the optimal control law for that case. The closest work to our presentation is [22]. Since we are motivated by a concrete problem of controlling a network of agents, we come up with stronger results. More importantly, we come up with a way to obtain the initial guess for numerical algorithms, which was identified as the major problem in [22].

The outline of the paper is as follows. We address a few mathematical preliminaries in the next section. Then we set up and solve the constrained controller synthesis problem. Then we show how this can be applied to the specific case of controlling a network of autonomous agents. We see that the specific structure of the problem can be exploited to yield simplifications. Then we present an example to illustrate the concepts and the method. We end with conclusions and present some avenues for further work.

\section{Mathematical Preliminaries And Notations}

By a network of interconnected dynamic agents, we mean a system of agents whose dynamics are not coupled but in which every agent can use the information from a prescribed 
set of other agents (called its out-neighbors) for calculating its control input. The flow of information is thus described by identifying the set of out-neighbors for each agent and is referred to as the information flow topology. Consider a network of $n$ agents. Together with the information flow topology, the network can be represented by a graph in which the agents are vertices and allowed communication links are edges. We refer to the agents variously as vertices, nodes or vehicles and the network as a graph or a formation. We define the Laplacian of a graph as $L=D-A$, where $D$ is the degree matrix and $A$ is the adjacency matrix of the graph. Consider the system evolving in time according to the equation

$$
x[k+1]=F x[k] .
$$

We say that the system is (asymptotically) stable, or that $F$ is (asymptotically) stable, if $F$ has all its eigenvalues on or inside (strictly inside) the unit circle and all the eigenvalues on the unit circle are simple.

We denote the expectation of a random variable $X$ by $E[X]$. The covariance matrix of a random variable $X$ with zero mean is defined by $E\left[X X^{T}\right]$. It is always a positive semi-definite matrix.

\section{The Optimal Constrained Control LaW}

Consider the following system

$$
\begin{aligned}
x[k+1] & =A x[k]+B u[k] \\
u[k] & =F x[k],
\end{aligned}
$$

where the initial condition $x[0]$ is random and is Gaussian with zero mean and covariance $R_{0}$. In the above equations, $x[k] \in \mathbf{R}^{n}$ is the state of the system and $u[k] \in \mathbf{R}^{m}$ is the control input. We denote the covariance of the state at time step $k$ by $R_{k}$. Suppose we wish to find the control law $F$ that minimizes the cost function

$$
J=E\left[\sum_{k=0}^{\infty}\left\{x^{T}[k] Q x[k]+u^{T}[k] R u[k]\right\}\right],
$$

where $Q$ is positive definite while $R$ is positive semidefinite. This is the classical $L Q R$ design problem solved through the discrete-time algebraic Riccati equation.

Now suppose that we wish to additionally constrain the control law to lie within a space spanned by the basis vectors $\left\{\Phi_{j}, j=1,2, \ldots, N\right\}$. It is fairly obvious that the optimal constrained control law would not, in general, be the projection of the optimal control law on to the space we are interested in. This is reminiscent of the fact that the optimal causal estimate for a random variable is not the same as the projection of the general optimal estimate on to the causal sub-space [13]. Also note that requiring a priori that the controller be linear might be a non-trivial assumption. But this allows us to derive algorithms for solving the problem and leads to sharper results.

Assume that a $F$ exists in the required space, such that $A+B F$ is stable. Then for that $F$, we obtain from (1)

$$
J=E\left[x^{T}[0] P x[0]\right],
$$

where $P$ is defined by

$$
P=\sum_{i=0}^{\infty}\left((A+B F)^{T}\right)^{i}\left(Q+F^{T} R F\right)(A+B F)^{i} .
$$

It is apparent from the definition of $P$, that it satisfies the discrete algebraic Lyapunov equation (see, e.g., [9])

$$
P=\left(Q+F^{T} R F\right)+(A+B F)^{T} P(A+B F) .
$$

Thus the cost is given by $J=\operatorname{trace}\left(P R_{0}\right)$, where, as defined above, $R_{0}$ is the covariance of the initial condition $x[0]$. We will call the system stabilizable if there exists a control law in the desired space such that $A+B F$ is stable. Assuming that the system is stabilizable, we wish to find the scalar coefficients $\alpha_{1}, \alpha_{2}, \ldots, \alpha_{N}$ such that the control law

$$
F=\sum_{i=1}^{N} \alpha_{i} \Phi_{i}
$$

minimizes the cost $J=\operatorname{trace}\left(P R_{0}\right)$, where $P$ satisfies (2) and $A+B F$ is stable. By the properties of the Lyapunov equation, if $(A+B F)$ is stable, $P$ is positive semi-definite.

\section{A. The Case where Noise is Present}

Suppose that the system evolves as

$$
\begin{aligned}
x[k+1] & =A x[k]+B u[k]+w[k] \\
u[k] & =F x[k] .
\end{aligned}
$$

The additional term $w[k]$ is stationary white Gaussian noise with zero mean and covariance $R_{w}$. Since the random noise goes on till infinite time, the cost function considered in (1) will diverge. Thus we need to reconsider what we mean by optimizing the cost function here. Following [15], we consider the following cost function

$$
J=\lim _{i \rightarrow \infty} E\left[x^{T}[i] Q x[i]+u^{T}[i] R u[i]\right] .
$$

Due to the assumed stabilizability, this cost function does not depend on the initial condition $x[0]$. Then by a derivation similar to the one given above and using the fact that the control law $F$ is stabilizing, we can write $J=\operatorname{trace}\left(P R_{w}\right)$, where $P$ is defined as before by (2). So we see that this problem reduces to the formulation stated above if we redefine $R_{0}$ to be $R_{w}$. From now on, we will study only the original formulation, assuming that such re-definitions have been carried out.

\section{B. Finding a Necessary Condition for the Optimal Solution}

This sub-section is on the lines of the analysis presented in [22]. We wish to find $F=\sum_{i=1}^{N} \alpha_{i} \Phi_{i}$ such that trace $\left(P R_{0}\right)$ is minimized, where $P$ satisfies (2). For a critical point,

$$
\operatorname{trace}\left(\frac{\partial P}{\partial \alpha_{i}} R_{0}\right)=0, \forall i=1,2, \ldots, N .
$$

If we define

$$
\Sigma_{i}=\Phi_{i}^{T}\left[R F+B^{T} P(A+B F)\right],
$$


we obtain from (2) that trace $\left(\frac{\partial P}{\partial \alpha_{i}} R_{0}\right)$ equals

$$
\operatorname{trace}\left(\left((A+B F)^{T} \frac{\partial P}{\partial \alpha_{i}}(A+B F)+\Sigma_{i}+\Sigma_{i}^{T}\right) R_{0}\right) \text {. }
$$

Further using the fact that $\operatorname{trace}(A B)=\operatorname{trace}(B A)$, and that the covariance of the state at time $k, R_{k}$, evolves as

$$
R_{k+1}=(A+B F) R_{k}(A+B F)^{T},
$$

yields

$$
\operatorname{trace}\left((A+B F)^{T} \frac{\partial P}{\partial \alpha_{i}}(A+B F) R_{0}\right)=\operatorname{trace}\left(\frac{\partial P}{\partial \alpha_{i}} R_{1}\right) \text {. }
$$

Using the above relation $k$ times, we obtain

$$
\operatorname{trace}\left(\frac{\partial P}{\partial \alpha_{i}} R_{0}\right)=\operatorname{trace}\left(\frac{\partial P}{\partial \alpha_{i}} R_{k}+\Sigma_{i} X_{k}+\Sigma_{i}^{T} X_{k}\right)
$$

where

$$
X_{k}=R_{0}+R_{1}+\cdots+R_{k} .
$$

But if $(A+B F)$ is stable, $R_{k}$ would be approximately a zero matrix for sufficiently large values of $k$. Thus if we denote

$$
X=R_{0}+R_{1}+R_{2}+\ldots,
$$

so that $X$ satisfies the Lyapunov equation

$$
X=R_{0}+(A+B F) X(A+B F)^{T},
$$

the necessary condition for a critical point can be written as follows. We want

$$
\operatorname{trace}\left(\Sigma_{i} X+\Sigma_{i}^{T} X\right)=0, \forall i=1, \ldots, N,
$$

where $F=\sum_{i=1}^{N} \alpha_{i} \Phi_{i}, P$ satisfies (2), $\Sigma_{i}$ is defined by (3) and $X$ satisfies (4).

Note that if $\Phi_{i}$ denotes a matrix with all elements zero except the $\left(j_{i}, k_{i}\right)$ th element being unity; the necessary condition given in $(5)$ reduces to

$$
\left[\left(B^{T} P(A+B F)+R F\right) X\right]_{j_{i}, k_{i}}=0, \forall i=1,2, \ldots, N,
$$

where $[A]_{i j}$ denotes the $(i, j)$-th element of the matrix $A$. Thus in the particular case when $F$ has no restrictions on its structure we get back the usual condition

$$
B^{T} P(A+B F)+R F=0 .
$$

\section{A Gradient Descent Method}

One method to obtain the control law is to solve (5) iteratively. Alternatively, we can also use a gradient search algorithm for the minimization problem. The algorithm is given by

\section{1) Initialize:}

a) Start from an initial guess of the set $\left\{\alpha_{i}\right\}$.

b) Solve (2) for $P$ using this value of the control law.

c) Check if $P$ is positive semi-definite. If yes, proceed to the update step; else repeat initialization with another guess.

\section{2) Update:}

a) Solve for $\frac{\partial P}{\partial \alpha_{i}}$ using (2) and the set $\left\{\alpha_{i}\right\}$.

b) Calculate the cost trace $\left(P R_{0}\right)$ and the gradient of the cost $\operatorname{trace}\left(\frac{\partial P}{\partial \alpha_{i}} R_{0}\right)$.

c) Update the guess by changing the current guess $\left\{\alpha_{i}\right\}$ by some constant amount $\delta$ times the gradient of the cost function.

d) Resolve (2) for $P$ using this value of the control law.

e) i) Check if $P$ is positive semi-definite.

ii) Check if this value of $P$ reduces the cost.

iii) If both $\mathrm{i}$ and ii are true, proceed to the next step. Otherwise, reduce $\delta$ by a half and again try to update. If stuck on this step for a long time, declare minima reached and terminate.

f) Adopt the updated value of guess as the current guess and go through the update step again.

Note that there exist in the literature (e.g. [22]) sophisticated methods to generate the initial values of the control law which are stabilizing and satisfy the topological constraints. However, as indicated in the next section, for our application, there exists a particularly simple way to generate the initial guess. Also, we have presented a very elementary version of the gradient search algorithm. Ways to make it more efficient and fast can readily be thought of.

Convergence Issues: As discussed later, in our specific problem, there is always a way to generate an admissible initial guess. Thus the algorithm will always provide us with $a$ solution. Numerical evidence suggests that in our problem the algorithm converges to a global minimum; however we have not been able to prove it. In some special cases (say when $A, B$ and $Q$ are the identity matrices, $R$ is zero and $F$ is constrained to be symmetric, corresponding to one of the cases considered in [25]) the problem becomes a convex problem; hence the algorithm does converge to a global minimum.

\section{Additional Results}

We can consider the question whether we can at all control (or stabilize) the system with control laws in a particular space. The question reduces to whether we can control the zeros of the polynomial $\operatorname{det}(\lambda I-A-B F)$ by choosing $F$ appropriately. A sufficient condition can be derived by using the implicit function theorem (e.g., [17]) and is omitted for space constraints. Bounds on the cost function can also be derived by using the bounds on the solution of the discrete algebraic Lyapunov equation (e.g., [7], [14]).

\section{ApPlication to CONTROL OF INTERCONNECTED DYNAMIC AGENTS}

As discussed above, the motivating example for the constrained synthesis problem is synthesis of a control law for a formation of interconnected agents. In this section, we cast this problem into the framework discussed above and discuss the decentralized implementation of the control law. 
We assume that the topology of the network is fixed and given. Moreover the topology is known to all the agents. If that is not the case, we might need to run a broadcast algorithm to exchange the topology information among the nodes. We want the control law to be decentralized in the sense that it should respect the topology for the measurements available to each node. Thus no node should need to access the information not directly available to it from its neighbors. Further we assume that the links are ideal when they exist. Finally, for simplicity, we assume that the full state of the agents is observed and measured. The generalization to output feedback is easily done.

Suppose the $i$-th agent is described by

$$
X_{i}[k+1]=\Phi X_{i}[k]+\Gamma U_{i}[k],
$$

where the control law $U_{i}[k]$ is given by

$$
U_{i}[k]=F_{i}^{1} X_{i}[k]+\sum_{\text {all out-neighbors } j} F_{i j}^{2}\left(X_{i}[k]-X_{j}[k]\right) .
$$

The system now evolves according to

$$
\begin{aligned}
X[k+1] & =(I \otimes \Phi) X[k]+(I \otimes \Gamma) U[k] \\
U[k] & =\left(\operatorname{diag}\left(F_{i}^{1}\right)+L_{\mathrm{gen}}\right) X[k],
\end{aligned}
$$

where $X[k]$ is the system state obtained by stacking the states of individual agents, $I$ is identity matrix of suitable dimension and $\operatorname{diag}\left(F_{i}^{1}\right)$ is a block diagonal matrix with $F_{i}^{1}$ 's along the diagonal and zero matrices elsewhere. $L_{\text {gen }}$ is a generalization of the Laplacian matrix of the graph and is formed as follows. Create the adjacency matrix $A$ for the network. Then replace each unity element that is at the $(i, j)$-th place by $-F_{i j}^{2}$. Replace the diagonal element in the $i$-th row by a matrix which is the sum of all matrices $F_{i j}^{2}$ for fixed $i$ and all possible $j$. Rest of the zero elements are replaced by zero matrices of appropriate dimensions.

Thus it can be seen that the problem is within the framework considered in the previous section. We solve for the optimal control law pertaining to the following system

$$
\begin{aligned}
X[k+1] & =(I \otimes \Phi) X[k]+(I \otimes \Gamma) U[k] \\
U[k] & =F X[k],
\end{aligned}
$$

with the additional constraint that $F$ should have those elements as 0 which correspond to zero entries in the $L_{\text {gen }}$ of the interconnection topology formed as above. $F$ can then readily be cast in the form $\operatorname{diag}\left(F_{i}^{1}\right)+L_{\text {gen }}$ and the matrices $F_{i}^{1}$ and $F_{i, j}^{2}$ obtained.

\section{Remarks:}

1) It may be noted that since each node knows the topology of the network, it knows the space within which the matrix $F$ should lie. Thus every node can carry the calculation given above in parallel to obtain the optimal control law for the whole system. Then it can extract the control law that it needs to apply.

2) If all the vehicles are not identical, a similar procedure can be applied. The matrices $I \otimes \Phi$ and $I \otimes \Gamma$ will be replaced by block diagonal matrices $\operatorname{diag}\left(\Phi_{i}\right)$ and $\operatorname{diag}\left(\Gamma_{i}\right)$, but other details remain similar.

\section{A. Stabilizability}

We wish to answer the question if it is possible to stabilize a formation when the vehicles are individually not stable. We have the following result.

Theorem 1: Consider a formation of interconnected dynamic agents as defined in section II.

1) A formation is controllable if and only if each individual agent is controllable.

2) A formation is stabilizable if and only if each individual agent is stabilizable.

Proof: The proof is relatively straight-forward. We write the controllability matrices of the formation and of the agent and use the standard properties of Kronecker products. We readily obtain that the controllability matrix of the formation is full rank if and only if each individual agent is controllable. Further the subspace not spanned by the columns of the controllability matrix of the formation is the same as the uncontrollable subspace of the agents.

\section{Remarks:}

1) Note that the theorem holds for both identical and different agent dynamics as well.

2) In our case, this result is important since it can be utilized to generate the starting guess for the numerical algorithms presented before. We find the control law required by each vehicle to stabilize itself while using only its own information. The initial guess can always be the block diagonal matrix formed by stacking this law along the diagonal of a matrix. This will always be a control law which stabilizes the formation, yet satisfies the topological constraints. Thus we are rid of the problem of choosing initial guesses for the algorithms. As discussed in [22], generating suitable initial guesses for the general case is a non-trivial task.

\section{B. The Completely Decentralized Case}

We consider the case where no agent has access to measurements of states of other agents. This case is interesting since we can analytically calculate the optimal control law and evaluate the cost. Moreover, since we always allow absolute measurements of the vehicle's own state, the control matrix always has at least the diagonal elements non-zero. Thus a lower bound for the cost for any topology is given by the cost achievable for the completely decentralized case.

For simplicity, we consider the case for the agent dynamics being a single integrator. We denote the $(i, j)$-th element of matrix $P$ by $[P]_{i, j}$. If we let $\Phi_{i}$ be the matrix with zeros everywhere except the $(i, i)$-th element, we know that the optimal control law is of the form

$$
F=\sum_{i} \alpha_{i} \Phi_{i}
$$

such that $F$ satisfies

$$
[(h P(I+h F)+R F) X]_{i, i}=0, \forall i .
$$


The matrix $X$ satisfies the Lyapunov equation

$$
X=(I+h F) X(I+h F)^{T}+R_{0} .
$$

Assume that all the initial states are independent of each other; thus $R_{0}$ is a diagonal matrix. $X$ satisfies

$$
X=\sum_{k=0}^{\infty}(I+h F)^{k} R_{0}\left((I+h F)^{k}\right)^{T},
$$

and thus $X$ is also a diagonal matrix. The $(i, i)$-th element is given by

$$
[X]_{i, i}=\frac{\left[R_{0}\right]_{i, i}}{1-\left(1+h[F]_{i, i}\right)^{2}} .
$$

Note that as long as the initial cost is randomly chosen, no diagonal element of $X$ can be zero. The condition for optimal $F$ reduces to

$$
[h P(I+h F) X]_{i, i}+[R F X]_{i, i}=0,
$$

which, in turn, reduces to

$$
\left([h P(I+h F)]_{i, i}+[R F]_{i, i}\right)[X]_{i, i}=0 .
$$

Now since no diagonal element of $X$ is 0 , this yields

$$
[h P(I+h F)]_{i, i}+[R F]_{i, i}=0,
$$

or finally

$$
h[P]_{i, i}+h^{2}[P]_{i, i}[F]_{i, i}+[R]_{i, i}[F]_{i, i}=0 .
$$

But since $P$ is given by

$$
P=\sum_{k=0}^{\infty}\left((I+h F)^{T}\right)^{k}\left(F^{T} R F+Q\right)(I+h F)^{k},
$$

thus $[P]_{i, i}$ is calculated to be

$$
[P]_{i, i}=\sum_{k=0}^{\infty}\left(1+h[F]_{i, i}\right)^{2 k}\left[F^{T} R F+Q\right]_{i, i} .
$$

Thus only the diagonal terms of $Q$ and $R$ play a role. The element $[P]_{i, i}$ evaluates to

$$
[P]_{i, i}=\frac{\left([F]_{i, i}\right)^{2}[R]_{i, i}+[Q]_{i, i}}{1-\left(1+h[F]_{i, i}\right)^{2}} .
$$

The optimal control law and the resulting cost can be readily calculated. We see that if we do not allow the vehicles to talk to each other, the off-diagonal terms of the cost matrices do not matter. Moreover, in this case, the cost simply turns out to be the sums of the costs incurred in controlling individual vehicles using their own state measurements with the cost function using only the diagonal terms of the cost function matrices. Also note that this analysis holds for the cases of all vehicles not being the same and also for general plant dynamics where we talk about block diagonal matrices. However it breaks down if, e.g., the initial conditions are not all independent. As stated above, this analysis provides a lower bound for the cost incurred in any topology. Similarly the completely centralized case provides an upper bound for the cost achieved by any topology.

\section{Example}

We now consider an example to illustrate the issues involved. We use the dynamics of each agent as the dynamics of the Caltech Multi Vehicle Wireless Testbed vehicles, as described in [2], [21]. The non-linear dynamics are given by

$$
\begin{aligned}
m \ddot{x} & =-\mu \dot{x}+\left(F_{L}+F_{R}\right) \cos (\theta) \\
m \ddot{y} & =-\mu \dot{y}+\left(F_{L}+F_{R}\right) \sin (\theta) \\
J \ddot{\theta} & =-\psi \dot{\theta}+\left(F_{R}-F_{L}\right) r_{f} .
\end{aligned}
$$

$F_{L}$ and $F_{R}$ are the inputs, $m=0.749 \mathrm{~kg}$ is the mass of vehicle, $J=0.0031 \mathrm{~kg} \mathrm{~m}^{2}$ is the moment of inertia, $\mu=0.15$ $\mathrm{kg}-\mathrm{s}$ is the linear frictional coefficient, $\psi=0.005 \mathrm{kgm}^{2} / \mathrm{s}$ is the rotational friction coefficient and $r_{f}=0.089 \mathrm{~m}$ is the distance from the center of mass of the vehicle to the axis of the fan. On linearizing the dynamics about the straight line $y=x$ at a velocity of $1 \mathrm{~ms}^{-1}$ along the $\mathrm{x}$ and $\mathrm{y}$ axes, we obtain the linearized dynamics of each agent described according to

$$
\begin{array}{ll}
\dot{X}=A X+B U & U=F X \\
X=\left[\begin{array}{llllll}
x & y & \theta & \dot{x} & \dot{y} & \dot{\theta}
\end{array}\right]^{T} \quad U=\left[\begin{array}{lll}
F_{L} & F_{R}
\end{array}\right]^{T}
\end{array}
$$

$$
A=\left[\begin{array}{cccccc}
0 & 0 & 0 & 1 & 0 & 0 \\
0 & 0 & 0 & 0 & 1 & 0 \\
0 & 0 & 0 & 0 & 0 & 1 \\
0 & 0 & \frac{-\left(F_{L}^{n}+F_{R}^{n}\right) \sin \left(\theta^{n}\right)}{m} & \frac{-\mu}{m} & 0 & 0 \\
0 & 0 & \frac{\left(F_{L}^{n}+F_{R}^{n}\right) \cos \left(\theta^{n}\right)}{m} & 0 & \frac{\mu}{m} & 0 \\
0 & 0 & 0 & 0 & 0 & \frac{-\psi}{J}
\end{array}\right]
$$

$$
\begin{aligned}
& B=\left[\begin{array}{lr}
0 & 0 \\
0 & 0 \\
0 & 0 \\
\frac{\cos \left(\theta^{n}\right)}{m} & \frac{\cos \left(\theta^{n}\right)}{m} \\
\frac{\sin \left(\theta^{n}\right)}{m} & \frac{\sin \left(\theta^{n}\right)}{\frac{m}{-r_{f}}} \\
\frac{-r_{f}}{J} & \frac{1}{J}
\end{array}\right] \\
& \theta^{n}=\frac{\pi}{4} \\
& F_{L}^{n}=F_{R}^{n}=\frac{\mu}{\sqrt{2}} .
\end{aligned}
$$

We discretize the above equations with a step size $h=0.2$. We consider 8 vehicles starting from an octagonal formation and consider the topologies possible as the communication radius of each vehicle is increased. It is apparent that by symmetry there are 5 distinct topologies possible, with each vehicle talking to $0,2,4,6$ and 7 other vehicles respectively. The initial covariance matrix $R_{0}$ is the identity matrix. The cost function matrix $R$ is also identity while the matrix $Q$ is randomly generated. A typical curve for the varying of the costs as the communication radius is increased is given in figure 1.

Following general conclusions can be drawn for the example from the plot.

1) As more and more communication is allowed, the cost goes down. 


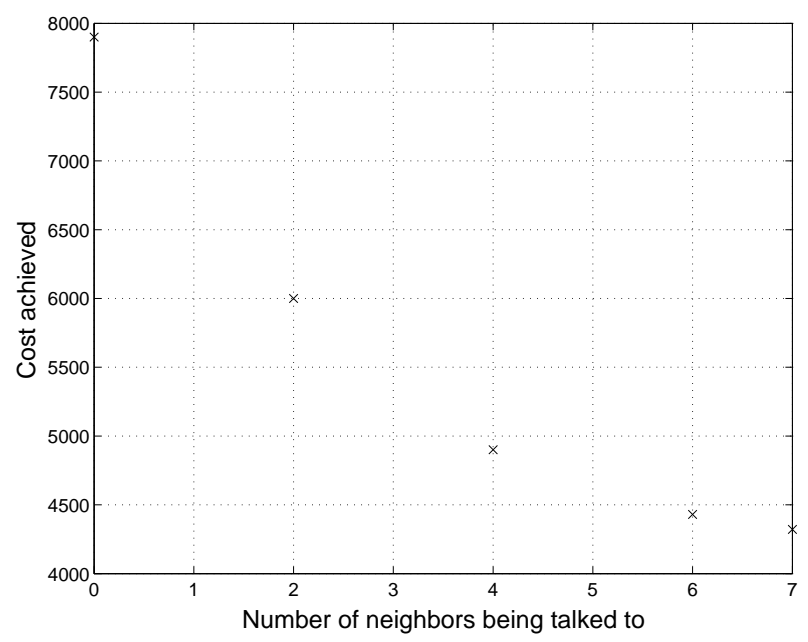

Fig. 1. As the communication radius is increased, the cost goes down.

2) The marginal utility of each communication link decreases as more and more links are added. However this might simply be due to the fact that the edges added later bring the data of far-away vehicles which is not so important for stabilization.

\section{Conclusions And Future Work}

In this paper, motivated by the problem of synthesis of optimal control laws for interconnected network of agents, we considered the synthesis of a LQR optimal control law which is constrained to lie in a particular space. We presented equations which can be iteratively solved to obtain the desired control law or used in a simple gradient descent algorithm for optimization. We saw that for the networked autonomous agents problem the numerical algorithms are free from the initial guess issues which are a big hurdle in the general problem. We discussed the implementation of the control laws so obtained in a decentralized fashion for the formation problem. We presented an example which illustrated the intuitive fact that communication in general helps to bring down the cost.

The work can potentially be extended in many ways. The most obvious direction is to consider the case when the nodes do not have an accurate or complete knowledge of the entire network. It would be interesting if we could evaluate the cost if the nodes utilize the information corresponding only to the neighboring nodes. Another avenue is to look at the issue of optimal topology. In general, the optimal cost is achieved by the fully centralized topology which is equivalent to every vehicle talking to every other vehicle. We might, however, be interested in putting additional constraints on the topology such as limiting the total number of links for reasons of congestion. Assuming identical vehicles and a symmetric cost function, a brute-force search on nonisomorphic graphs (see, e.g., [11]) is sufficient, rather than on all graphs on $n$-vertices. Even though that represents a huge reduction in number of graphs to be searched (e.g., for 5 vertices, we need to search only 34 graphs out of a possible 1024), a method better than brute-force search is desirable for large problems.

\section{REFERENCES}

[1] B. Bamieh, F. Paganini, and M. Dahleh. Optimal Control of Distributed Arrays with Spatial Invariance, volume 245 of Lecture Notes in Control and Information Sciences, pages 329-343. SpringerVerlag, 1999.

[2] Lars Cremean, William Dunbar, David van Gogh, Jason Hickey, Eric Klavins, Jason Meltzer, and Richard M. Murray. The Caltech multivehicle wireless testbed. In Proc of the 2002 Conference on Decision and Control, 2002.

[3] R. D'Andrea. A linear matrix inequality approach to decentralized control of distributed parameter systems. In Proc. of American Control Conference, 1998.

[4] R. D'Andrea, G. Dullerud, and S. Lall. $l_{2}$ controller synthesis for multidimensional systems. In Proc. of IEEE Conf. on Decision and Control, 1998.

[5] G. A. de Castro. Convex Methods for the design of Structured Controllers. PhD thesis, University of California, Los Angeles, 2003.

[6] C. Fan, J. L. Speyer, and C. R. Jaensch. Centralized and decentralized solutions of the linear-exponential-gaussian problem. IEEE Transactions on Automatic Control, 39(10):1986-2003, 1994.

[7] Y. Fang, K. A. Loparo, and X. Feng. Inequalities for the trace of matrix product. IEEE Transactions on Automatic Control, 39(1):5557, 1987.

[8] J. A. Fax. Optimal and Cooperative Control of Vehicle Formations. $\mathrm{PhD}$ thesis, California Institute of Technology, 2001.

[9] Z. Gajic and M. T. J. Qureshi. Lyapunov Matrix Equation in System Stability and Control. Academic Press, Inc, 1995.

[10] V. Gupta, B. Hassibi, and R. M. Murray. Stability analysis of stochastically varying formations of dynamic agents. In Proc. of IEEE Conf. on Decision and Control, 2003.

[11] F Harary. Graph Theory. Addison-Wesley, 1994.

[12] A. Jadbabaie, J. Lin, and A. S. Morse. Coordination of groups of mobile autonomous agents using nearest neighbor rules. IEEE Transactions on Automatic Control, 48(6):988-1001, 2003.

[13] T. Kailath, A. H. Sayed, and B. Hassibi. Linear Estimation. Prentice Hall, 2000.

[14] N. Komaroff. Upper bounds for the eigenvalues of the solution of the discrete algebraic Lyapunov equation. IEEE Transactions on Automatic Control, 35(4):468-469, 1990.

[15] H. Kwakernaak and R. Sivan. Linear Optimal Control Systems. John Wiley and Sons, 1972.

[16] W. S. Levine, T. L. Johnson, and M. Athans. Optimal limited state variable feedback controllers for linear systems. IEEE Transactions on Automatic Control, AC-16:785-793, 1971.

[17] J. E. Marsden and M. Hoffman. Elementary Classical Analysis. W. H. Freeman and co., 2 edition, 1993.

[18] C. H. Papadimitriou and J. N. Tsitsiklis. Intractable problems in control theory. SIAM Journal on Control and Optimization, 24:639654, 1986.

[19] M. Rotkowitz and S. Lall. Decentralized control information structures preserved under feedback. In Proc. of IEEE Conf. on Decision and Control, 2002.

[20] T. Soderstrom. On some algorithms for design of optimal constrained regulators. IEEE Transactions on Automatic Control, AC-23:11001101, 1978.

[21] S. Waydo, Z. Jin, E. Wildanger, M. Lammers, H. Scholze, P. Foley, R. M. Murray, and D. Held. MVWT-II: The second generation caltech multi-vehicle wireless testbed. In American Control Conference, 2004. To Appear.

[22] C. J. Wenk and C. H. Knapp. Parameter optimization in linear systema with arbitrarily constrained controller structure. IEEE Transactions on Automatic Control, AC-25(3):496-500, 1980.

[23] H. S. Witsenhausen. A counterexample in stochastic optimum control. SIAM Journal of Control, 6(1):131-147, 1968.

[24] H. S. Witsenhausen. Separation of estimation and control for discrete time systems. Proceedings of the IEEE, 59(11):1557-1566, 1971.

[25] L. Xiao and S. Boyd. Fast linear iterations for distributed averaging. In Proc. of the 42nd IEEE Conference on Decision and Control, pages 4997-5002, 2003. 